\title{
ON THE PROBABILITY DISTRIBUTION OF THE OPTIMUM OF A RANDOM LINEAR PROGRAM*
}

\author{
András Prékopa \\ Eötvös L. University, Budapest, Hungary \\ and \\ the Mathematical Institute of the Hungarian Academy of Sciences
}

\section{Introduction}

In the present paper we shall consider linear programming problems

$$
\begin{aligned}
\mu & =\max c^{\prime} x \\
A x & =b, \quad x \geq 0,
\end{aligned}
$$

where $A$ is an $m \times n$ matrix, $c$ and $x$ are $n$-dimensional vectors, and $b$ is an $m$-dimensional vector. We shall suppose that $A, b, c$ have random elements and components, respectively. As $\mu$ is a function of the variables in $A, b$ and $c$,

$$
\mu=\mu(A, b, c)
$$

it is also a random variable and its probability distribution is what we are interested in. This problem is of basic importance and is conceivable as a stochastic sensitivity analysis of a linear programming model. The question how the transformation $A, b, c \rightarrow \mu$ operates under the presence of random influences in $A, b$, and $c$ does not play just the role of a sensitivity analysis, however. In fact, in $A, b, c$ we may have not just small random disturbances but random variables of significant variation.

The problem in its general form has been considered by Tintner [2], [3], and BABBAR [1]. In these papers it is supposed that the random variation does not change the optimal basis in the sense that the subscripts of the optimal basis vectors remain the same for all possible values of $A, b, c$. Thus finding the probability distribution of $\mu$ is equivalent to finding the probability distribution of an (also random) linear functional defined over the random solution of a set of linear equations. In this respect it is also possible to proceed

\footnotetext{
${ }^{*}$ Received by the editors July 12, 1965. Presented at the First International Conference on Programming and Control, held at the United States Air Force Academy, Colorado, April 15, 1965.
} 
in two different ways: either to develop $\mu$ into a finite Taylor series and use the leading, linear terms as an approximation to $\mu$ and obtain its probability distribution, or to consider the components of the solution as fractions of random determinants, approximate their distributions by the normal law and again approximate by the normal law the fraction of two normally distributed variables. This method has the handicap that it produces sophisticated approximation formulas.

In $\S 2,3,4$, we consider systems of linear equations, the probability distribution of a random linear functional defined over the solutions and apply this theory for our original problem concerning random linear programs. Our approximation formulas for the characteristics of $\mu$, especially for the dispersion, will be particularly simple, as simple as possible in this general formulation of the problem from the point of view of practical application, involving the primal and dual optimal solutions of the linear programming problem carried out with the expectations and the covariances of the present random variables. We express our statements in limit theorems and list carefully all mathematical assumptions. Our results are formulated in general, containing the essential features of the problem and allowing the possibility of specialization when facing a particular problem.

The results of the present paper, however, apply to the case when the random elements in the technology matrix keep the optimal basis (basis subscripts), obtained by computing with the expectations, with a high probability. To more general questions we return in subsequent papers.

Since in the present approach the principal aim is to reduce $\mu$ to a sum of random variables, the asymptotic normality of this sum will be supposed. In the particular cases where the sum in question contains an increasing number of independent random variables, e.g., $A$ has independent elements or it is enough if its rows or columns are independent, the limit distribution theory of sums of double sequences of independent random variables can be applied. (See [19].) If independence does not occur then we may suppose the joint normality of the random variables in $A, b, c$ which is sufficient, or suppose simply the sum in question to be normally distributed; but there is no detailed general theory of the limit distributions of sums of double sequences of dependent random variables. On the other hand, any particular problem reveals, in some specific way, how the random elements intervene, from the knowledge of which we may assume the normality of the approximating sum.

\section{Systems of random linear equations}

Consider the following system of linear equations:

$$
\sum_{k=1}^{m} a_{i k} x_{k}=b_{i}, \quad i=1, \ldots, m .
$$

Let us denote by $B$ the matrix of the equations and by $b$ the vector consisting of the $b_{i}$ 's as components and let us introduce an $m$-dimensional vector $c$. If $B$ is nonsingular then 
(2.1) has a unique solution $B^{-1} b$. Suppose now that $B, c, b$ are all random and that all elements and components have finite variances. We are interested in the probability distribution of the functional

$$
\mu=c^{\prime} R b, \quad \text { where } R=B^{-1}
$$

and where the prime denotes transpose. In order to avoid complications in the notation we shall suppose that $B$ is independent of the couple $c, b$ and that $c, b$ are also independent of each other. This assumption does not play any significant role here. We shall denote by $a_{1}, \ldots, a_{m}$ the columns of $B$ and by $D_{i k}$ the cross covariance matrix of $a_{i}$ and $a_{k}$, i.e.,

$$
D_{i k}=\mathrm{E}\left[\left(a_{i}-a_{i}^{(0)}\right)^{\prime}\left(a_{k}-a_{k}^{(0)}\right)\right], \quad i, k=1, \ldots, m,
$$

where $a_{i}^{(0)}=\mathrm{E}\left(a_{i}\right), i=1, \ldots, m$, and $\mathrm{E}$ is the expectation operator. The expectations of $B, b, c, a_{i k}, b_{i}, c_{j}$ will be denoted by $B_{0}, b_{0}, c_{0}, a_{i k}^{(0)}, b_{i}^{(0)}, c_{j}^{(0)}$, respectively. $R_{0}$ will denote $B_{0}^{-1}$. The covariance matrices of $\gamma$ and $\beta$ will be denoted by $C$ and $F$, respectively.

Disregarding for a while the random nature of our quantities, we shall give the finite Taylor expansion of $\mu$ around the expectations, as far as the second order terms. It can be done by using a formula well-known in matrix theory stating that if the inverse of a nonsingular square matrix is $R$ and we modify the original matrix by adding $\xi$ to the element in the $i$ th row and $k$ th column then the inverse of the modified matrix will be

$$
R-\frac{\xi}{1+r_{k i} \xi}\left(\begin{array}{c}
r_{1 i} \\
\vdots \\
r_{m i}
\end{array}\right)\left(r_{k 1} \cdots r_{k m}\right) \text {. }
$$

Hence if we change $B$ in the manner described above and consider the change in the functional then we obtain

$$
\mu\left(a_{i k}+\xi\right)-\mu\left(a_{i k}\right)=-\frac{\xi}{1+r_{k i} \xi} y_{i} x_{k}
$$

where

$$
y_{i}=\sum_{j=1}^{m} c_{j} r_{j i}
$$

From this it follows that

$$
\frac{\partial \mu}{\partial a_{i k}}=y_{i} x_{k}, \quad i, k=1, \ldots, m .
$$

By a double application of $(2.4)$ we get

$$
\mu\left(a_{i k}+\xi\right)+\mu\left(a_{i k}-\xi\right)-2 \mu\left(a_{i k}\right)=\xi^{2} y_{i} x_{k} \frac{r_{k i}}{1-\xi^{2} r_{k i}^{2}},
$$


hence

$$
\begin{aligned}
\frac{\partial^{2} \mu}{\partial a_{i k}^{2}} & =\lim _{\xi \rightarrow 0} \frac{\mu\left(a_{i k}+\xi\right)+\mu\left(a_{i k}-\xi\right)-2 \mu\left(a_{i k}\right)}{\xi^{2}} \\
& =y_{i} x_{k} r_{k i}, \quad i, k=1, \ldots, m .
\end{aligned}
$$

We can determine similarly the mixed partial derivatives. The result is the following

$$
\begin{aligned}
\frac{\partial^{2} \mu}{\partial a_{i k} \partial a_{p q}} & =\lim _{\substack{\xi \rightarrow 0 \\
\eta \rightarrow 0}} \frac{\mu\left(a_{i k}+\xi, a_{p q}+\eta\right)-\mu\left(a_{i k}+\xi, a_{p q}\right)-\mu\left(a_{i k}, a_{p q}+\eta\right)+\mu\left(a_{i k}, a_{p q}\right)}{\xi \eta} \\
& =x_{k} y_{p} r_{q i}+x_{q} y_{i} r_{k p}, \quad \text { for } \quad|i-p|+|k-q|>0 .
\end{aligned}
$$

Let us finally mention the derivatives where $c_{i}$ and $b_{i}$ are involved:

$$
\begin{aligned}
\frac{\partial \mu}{\partial c_{j}} & =x_{j}, \quad \frac{\partial \mu}{\partial b_{j}}=y_{j}, \quad \frac{\partial^{2} \mu}{\partial c_{i} \partial b_{j}}=r_{i j}, \\
\frac{\partial^{2} \mu}{\partial a_{i k} \partial c_{j}} & =x_{k} r_{j i}, \quad \frac{\partial^{2} \mu}{\partial a_{i k} \partial b_{j}}=y_{i} r_{k j}, \quad i, j, k=1, \ldots, m .
\end{aligned}
$$

Let us introduce the notations

$$
\begin{aligned}
y^{\prime}=c^{\prime} R, & y_{0}^{\prime}=c_{0}^{\prime} R_{0}, \\
x=R b, & x_{0}=R_{0} b_{0},
\end{aligned}
$$

and denote by $x_{i}^{(0)}, y_{j}^{(0)}$ the components of $x_{0}, y_{0}$, respectively. Furthermore

$$
\begin{aligned}
a_{i k}-a_{i k}^{(0)} & =\xi_{i k}, & B-B_{0} & =\Xi, \\
c_{i}-c_{i}^{(0)} & =\gamma_{i}, & c-c_{0} & =\gamma, \\
b_{i}-b_{i}^{(0)} & =\beta_{i}, & b-b_{0} & =\beta, \quad i, k=1, \ldots, m .
\end{aligned}
$$

With the aid of these notations the desired Taylor expansion is the following:

$$
\begin{aligned}
\mu= & \mu_{0}-\sum_{i, k=1}^{m} y_{i}^{(0)} \xi_{i k} x_{k}^{(0)}+\sum_{i=1}^{m} y_{i}^{(0)} \gamma_{i}+\sum_{k=1}^{m} x_{k}^{(0)} \beta_{k} \\
& +\frac{1}{2} \sum_{i, k=1}^{m} y_{i} \xi_{i k}^{2} r_{k i} x_{k}+\sum_{i, k, j=1}^{m} x_{k} r_{j i} \xi_{i k} \gamma_{j} \\
& +\sum_{i, k, j=1}^{m} y_{i} r_{k j} \xi_{i k} \beta_{j}+\sum_{|i-p|+|k-q|>0}\left(x_{k} y_{p} r_{q i}+x_{q} y_{i} r_{k p}\right) \xi_{i k} \xi_{p q},
\end{aligned}
$$

or in a concise form,

$$
\mu-\mu_{0}=-y_{0}^{\prime} \Xi x_{0}+y_{0}^{\prime} \gamma+x_{0}^{\prime} \beta+\rho,
$$


where the error term $\rho$ is given by

$$
\rho=-\frac{3}{2} x^{\prime} \Xi_{2} y+2 y^{\prime} \Xi R \Xi x+\gamma^{2} R \Xi x+y^{\prime} \Xi R \beta,
$$

and $\Xi_{2}$ is the matrix consisting of the entries $\xi_{i k}^{2} r_{k i}$. In the above development of the error term, $x, y$, and $R$, which are functions of $b, c, B$, are taken at a point $b_{i}^{(0)}+\vartheta \beta_{i}, c_{i}^{(0)}+\vartheta \gamma_{i}$, $a_{i j}^{(0)}+\vartheta \xi_{i j}$, where $0<\vartheta<1$.

The leading term in (2.14) has expectation 0 and variance

$$
\sigma^{2}=\sum_{i, k=1}^{m} x_{i}^{(0)} y_{0}^{\prime} D_{i k} y_{0} x_{k}^{(0)}+y_{0}^{\prime} C y_{0}+x_{0}^{\prime} F x_{0} .
$$

If the columns of $B$ are independent random vectors, as it can be supposed in some practical cases, then $\sigma^{2}$ reduces to

$$
\sigma^{2}=\sum_{k=1}^{m}\left(x_{k}^{(0)}\right)^{2} y_{0}^{\prime} D_{k k} y_{0}+y_{0}^{\prime} C y_{0}+x_{0}^{\prime} F x_{0},
$$

which further reduces if all elements of $B$ are independent. If also $c$ and $b$ have independent components then we have

$$
\sigma_{2}=\sum_{i, k=1}^{m}\left(y_{i}^{(0)}\right)^{2} \sigma_{i k}^{2}\left(x_{k}^{(0)}\right)^{2}+\sum_{i=1}^{m}\left(y_{i}^{(0)}\right)^{2} s_{i}^{2}+\sum_{k=1}^{m}\left(x_{k}^{(0)}\right)^{2} t_{k}^{2},
$$

where

$$
\sigma_{i k}^{2}=\mathrm{E}\left(\xi_{i k}^{2}\right), \quad s_{i}^{2}=\mathrm{E}\left(\gamma_{i}^{2}\right), \quad t_{k}^{2}=\mathrm{E}\left(\beta_{k}^{2}\right), \quad i, k=1, \ldots, m .
$$

In the next sections we shall give sufficient conditions under which $\left(\mu-\mu_{0}\right) / \sigma$ has an asymptotic normal distribution. This will mean from the practical point of view that $\mu$ has an asymptotic normal distribution with expectation $\mu_{0}$, i.e., the value of the functional belonging to the expectations of all values involved, and variance given by (2.16) which may specialize.

\section{Limit distribution theorems for random linear equations}

First we prove a lemma.

Lemma Let $H_{1}, H_{2}, \ldots$ be a sequence of events with the property that

$$
\lim _{N \rightarrow \infty} \mathrm{P}\left(H_{N}\right)=1 .
$$

Let further $\eta_{N}$ and $\zeta_{N}$ be two sequences of random variables, where $\eta_{N}$ has a limit distribution, i.e.,

$$
\lim _{N \rightarrow \infty} \mathrm{P}\left(\eta_{N}<x\right)=G(x)
$$


at every point of continuity of $G(x)$ and $\zeta_{N}$ tends stochastically to 0 (in symbols, $\zeta_{N} \Rightarrow 0$ ), i.e.,

$$
\lim _{N \rightarrow \infty} \mathrm{P}\left(\left|\zeta_{N}\right|>\epsilon\right)=0, \quad \text { for every } \epsilon>0
$$

( $\zeta_{N}$ has a degenerate limit distribution). Under these conditions

$$
\lim _{N \rightarrow \infty} \mathrm{P}\left(\eta_{N}+\zeta_{N} \mid H_{N}\right)=G(x)
$$

at every point of continuity of $G(x)$.

Since this lemma is essentially Cramér's lemma (see [18, p. 254]) expressed in a slightly modified form, we omit the proof.

In order to obtain limit theorems we can proceed in two directions. We may keep $m$, the size of the system of equations, fixed, while the random disturbances have a slowing down tendency. This is the case when, for example, the random disturbances are due to some inaccuracy in the measurements of the data which shows a decreasing tendency upon using more data or, in other terms, a larger sample. The other possibility is to increase $m$. In this case we shall also suppose implicitly that the random elements are small as compared to the expectations, but for convergence to the normal distribution the increasing size of the matrix $B$ contributes also. First we formulate two theorems in general forms.

THEOREM 1 If a function $f\left(z_{1}, z_{2}, \ldots, z_{k}\right)$ has continuous second order derivatives in some convex neighborhood of the point $\left(z_{1}^{(0)}, z_{2}^{(0)}, \ldots, z_{k}^{(0)}\right)$, where $k$ is fixed, and if for a sequence of random vectors $\left(\xi_{1}^{(N)}, \xi_{2}^{(N)}, \ldots, \xi_{k}^{(N)}\right)$ with $\mathrm{E}\left(\xi_{i}^{(N)}\right)=0, i=1, \ldots, k, N=$ $1,2, \ldots$, the following conditions are satisfied:

$$
\begin{gathered}
\xi_{i}^{(N)} \Rightarrow 0 \quad \text { if } N \rightarrow \infty, \quad i=1, \ldots, k, \\
\lim _{N \rightarrow \infty} \mathrm{P}\left(\frac{1}{\sigma_{N}} \sum_{i=1}^{k} \frac{\partial f^{(0)}}{\partial z_{i}} \xi_{i}^{(N)}<x\right)=G(x),
\end{gathered}
$$

at every point of continuity of $G(x)$, where $\partial f^{(0)} / \partial z_{i}$ means the derivative $\partial f / \partial z_{i}$, taken at $\left(z_{1}^{(0)}, z_{2}^{(0)}, \ldots, z_{k}^{(0)}\right)$ and $\sigma_{N}$ is the dispersion of

$$
\begin{gathered}
\sum_{i=1}^{k}\left(\partial f^{(0)} / \partial z_{i}\right) \xi_{i}^{(N)}, \\
\frac{1}{\sigma_{N}} \sum_{i, j=1}^{k} \frac{\partial^{2} f^{(1)}}{\partial z_{i} \partial z_{j}} \xi_{i}^{(N)} \xi_{j}^{(N)} \Rightarrow 0 \text { if } N \rightarrow \infty,
\end{gathered}
$$

where the superscript (1) means that the derivative is taken at an arbitrary point of the convex domain and this point may also vary uith $N$, then we have

$$
\lim _{N \rightarrow \infty} \mathrm{P}\left\{\frac{1}{\sigma_{N}}\left[f\left(z_{1}^{(0)}+\xi_{1}^{(N)}, \ldots, z_{k}^{(0)}+\xi_{k}^{(N)}\right)-f\left(z_{1}^{(0)}, \ldots, z_{k}^{(N)}\right)\right]<x\right\}=G(x) .
$$


Proof. Let $H_{N}$ denote the event that $\left(z_{1}^{(0)}+\xi_{1}^{(N)}, \ldots, z_{k}^{(0)}+\xi_{k}^{(N)}\right)$ is in that neighborhood of $\left(z_{1}^{(0)}, \ldots, z_{k}^{(0)}\right)$ where $f$ has continuous second order derivatives. In this case,

$$
\begin{gathered}
f\left(z_{1}^{(0)}+\xi_{1}^{(N)}, \ldots, z_{k}^{(0)}+\xi_{k}^{(N)}\right)-f\left(z_{1}^{(0)}, \ldots, z_{k}^{(0)}\right) \\
=\sum_{i=1}^{k} \frac{\partial f^{(0)}}{\partial z_{i}} \xi_{i}^{(N)}+\frac{1}{2} \sum_{i, j=1}^{k} \frac{\partial^{2} f^{(1)}}{\partial z_{i} \partial z_{j}} \xi_{i}^{(N)} \xi_{j}^{(N)}
\end{gathered}
$$

where $\partial^{2} f^{(1)} / \partial z_{i} \partial z_{j}$ is the second order derivative taken at $\left(z_{1}^{(0)}+\vartheta \xi_{1}^{(N)}, \ldots, z_{k}^{(0)}+\vartheta \xi_{k}^{(N)}\right)$, $0<\vartheta<1$. According to $(1), \lim _{N \rightarrow \infty} \mathrm{P}\left(H_{N}\right)=1$. Let us divide by $\sigma_{N}$ on both sides in (3.1). Then the second term on the right-hand side tends stochastically to 0 according to (3). Let us denote this term by $\zeta_{N}$ and the first term by $\eta_{N}$. Then a direct application of the lemma completes the proof.

Condition (3) is clearly fulfilled if

$$
\frac{1}{\sigma_{N}} \xi_{i}^{(N)} \xi_{j}^{(N)} \Rightarrow 0, \quad i, j=1, \ldots, k
$$

Before stating Theorem 2, we mention the notion of a star domain. An open domain $K$ around and containing a point $\left(z_{1}, \ldots, z_{k}\right)$ in the $k$-dimensional space is called a star domain if the intersection of $K$ with any ray $\left(z_{1}+t \xi_{1}, \ldots, z_{k}+t \xi_{k}\right), t>0$, is an open interval. This may contain, in particular, every point of the ray. The point $\left(z_{1}, \ldots, z_{k}\right)$ is called the seed of the domain. This notion will be important to extend the possibility of the Taylor-series expansion around the given point as large as possible.

For later purposes we introduce a notion, that of a maximal star domain around a nonsingular matrix $B_{0}$, which by definition consists of all matrices of the form

$$
B_{0}+t \Xi
$$

where for any given $\Xi, t$ runs continuously from 0 until the sum becomes singular. That singular matrix is excluded, however.

THEOREM 2 Suppose that we have a sequence of functions of an increasing number of variables $f_{N}\left(z_{1}, z_{2}, \ldots, z_{k_{N}}\right)$ where $k_{N} \rightarrow \infty$ as $N \rightarrow \infty$, and each $f_{N}$ has a neighborhood, a star domain around a point $\left(z_{N 1}^{(0)}, \ldots, z_{N k_{N}}^{(0)}\right)$ where its second order derivatives exist and are continuous. Suppose furthermore that we have a double sequence of random variables $\xi_{1}^{(N)}, \xi_{2}^{(N)}, \ldots, \xi_{k_{N}}^{(N)}$, with expectations 0 and finite variances, satisfying the following conditions

$$
\left.\lim _{N \rightarrow \infty} \mathrm{P}\left\{z_{N 1}^{(0)}+\xi_{1}^{(N)}, \ldots, z_{N k_{N}}^{(0)}+\xi_{k_{N}}^{(N)}\right) \in K_{N}\right\}=1
$$

where $K_{N}$ is the above mentioned neighborhood,

$$
\lim _{N \rightarrow \infty} \mathrm{P}\left(\frac{1}{\sigma_{N}} \sum_{i=1}^{k_{N}} \frac{\partial f_{N}^{(0)}}{\partial z_{i}} \xi_{i}^{(N)}<x\right)=G(x)
$$


at every point of continuity of $G(x)\left(\partial f_{N}^{(0)} / \partial z_{i}\right.$, and $\partial^{2} f_{N}^{(1)} / \partial z_{i} \partial z_{j}$ have the same meaning as in Theorem 1),

$$
\frac{1}{\sigma_{N}} \sum_{i, j=1}^{k_{N}} \frac{\partial^{2} f_{N}^{(1)}}{\partial z_{i} \partial z_{j}} \xi_{i}^{(N)} \xi_{j}^{(N)} \Rightarrow 0 \text { if } N \rightarrow \infty
$$

Then

$$
\lim _{N \rightarrow \infty} \mathrm{P}\left\{\frac{1}{\sigma_{N}}\left[f_{N}\left(z_{N 1}^{(0)}+\xi_{1}^{(N)}, \ldots, z_{N k_{N}}^{(0)}+\xi_{k_{N}}^{(N)}\right)-f\left(z_{N 1}^{(0)}, \ldots, z_{N k_{N}}^{(0)}\right)\right]<x\right\}=G(x)
$$

at every point of continuity of $G(x)$.

The proof is similar to that of Theorem 1.

It is worth mentioning that the fulfillment of condition (1) in Theorem 2 may be the cause of the slowing down tendency of random elements or the increase of $K_{N}$ or both.

In both theorems we used the same idea Cramér used when proving the asymptotic normality of functions of moments (see [19, pp. 366-367], noting that in that case the number of variables is fixed). We can apply these theorems for random linear equations. In the following theorem we shall omit the subscript $N$ which would refer to the fact that we have a sequence of random elements. Thus all our previous notations concerning random equations are applicable.

Theorem 3 Suppose that $m, B_{0}, c_{0}, b_{0}$ are fixed and that $B_{0}$ is nonsingular and introduce the following conditions:

$$
\begin{gathered}
\sigma_{i k} \rightarrow 0, \quad i, k=1, \ldots, m, \\
\mathrm{P}\left\{\frac{1}{\sigma}\left[-y_{0}^{\prime} \Xi x_{0}+y_{0}^{\prime} \gamma+x_{0}^{\prime} \beta\right]<x\right\} \rightarrow \Phi(x)=\frac{1}{\sqrt{2 \pi}} \int_{-\infty}^{x} e^{-u^{2} / 2} \mathrm{~d} u, \\
\text { for every } x,-\infty<x<\infty \\
\frac{\rho}{\sigma} \Rightarrow 0 .
\end{gathered}
$$

Then for every $x$,

$$
\mathrm{P}\left(\frac{\mu-c_{0}^{\prime} R_{0} b_{0}}{\sigma}<x\right) \rightarrow \Phi(x)
$$

Proof. Theorem 3 is an immediate consequence of Theorem 1 applied to the function $\mu=\mu(A, b, c)$ of $m^{2}+2 m$ variables, $\left(A_{0}, b_{0}, c_{0}\right)$ as the point around which the Taylor-series development is taken, and $\Xi, \gamma, \beta$ as the sequence of $\left(m^{2}+2 m\right)$-dimensional random vectors. We just have to mention that condition (1) in Theorem 1 is ensured by condition (1) of Theorem 3. Various consequences of this theorem can be derived. Among them we mention the simplest. 
Corollary Suppose that the $m^{2}+2 m$ random variables in $\Xi$, $\gamma$ and $\beta$ have a normal joint distribution and

$$
\sigma_{\max } \rightarrow 0, \quad \frac{\sigma_{\max }^{2}}{\sigma} \rightarrow 0, \quad \text { where } \sigma_{\max }=\max \left(\sigma_{i k}, s_{i}, t_{k}\right)
$$

Then (3.2) holds.

Proof. All that we have to verify is the fulfillment of (3) in Theorem 3. If we look at the detailed expression of $\rho$ given by the last terms in (2.13), we see that, separately, each term of that sum divided by $\sigma$ converges stochastically to 0 . In fact, considering the quadratic terms $\xi_{i k}^{2} / \sigma$ we see by the Markov inequality that

$$
\mathrm{P}\left(\frac{\xi_{i k}^{2}}{\sigma}>\epsilon\right) \leq \frac{\mathrm{E}\left(\xi_{i k}^{2}\right)}{\sigma} \leq \frac{\sigma_{\max }^{2}}{\sigma} \rightarrow 0 .
$$

For all other terms the Chebyshev inequality can be applied.

THEOREM 4 Consider a sequence of matrices and vectors $B_{0}, c_{0}, b_{0}$, and a corresponding random sequence $\Xi, \gamma, \beta$ (the subscripts are omitted), where $m$, the size of the matrices (equal to the dimension of the vectors), tends to infinity. Suppose that all $B_{0}$ matrices are nonsingular. To every $B_{0}$ in the sequence there corresponds a maximal star domain $K$ where $B_{0}+\Xi$ is nonsingular and the Taylor expansion around $B_{0}$ applies. Suppose that

$$
\begin{gathered}
\mathrm{P}\left(B_{0}+\Xi \in K\right) \rightarrow 1, \\
\mathrm{P}\left\{\frac{1}{\sigma}\left(-y_{0}^{\prime} \Xi x_{0}+x_{0}^{\prime} \gamma+y_{0}^{\prime} \beta\right)<x\right\} \rightarrow \Phi(x), \quad-\infty<x<\infty, \\
\frac{\rho}{\sigma} \Rightarrow 0 .
\end{gathered}
$$

Under these conditions ${ }^{1}$

$$
\mathrm{P}\left\{\frac{1}{\sigma}\left(\mu-c_{0}^{\prime} R_{0} b_{0}\right)<x\right\} \rightarrow \Phi(x), \quad-\infty<x<\infty .
$$

The proof of this theorem is similar to that of Theorem 3. Analyzing the conditions here, (1) and (2) are realistic as the size of the matrix increases. The crucial point is condition (3) which may very easily fail to hold. In fact, first of all, the fourth term containing the squares $\xi_{i k}^{2}$ may not be negligible as compared to $\sigma$. It does not have, in general, expectation 0 even in the case where all random variables are independent. It seems, therefore, advisable to attach the sum

$$
\frac{1}{2} \sum_{i, k=1}^{m} y_{i}^{(0)} \xi_{i k}^{2} r_{k i}^{(0)} x_{k}^{(0)}
$$

\footnotetext{
${ }^{1}$ Instead of $\Phi(x)$ we may suppose some other distribution function too.
} 
to the leading term, changing it into

$$
-\sum_{i, k=1}^{m} y_{i}^{(0)} \xi_{i k}\left(1-\frac{1}{2} \xi_{i k} r_{k i}\right) x_{k}^{(0)}+y_{0}^{\prime} \gamma+x_{0}^{\prime} \beta .
$$

We may then approximate the distribution of $\mu$ by a normal distribution with the expectation

$$
c_{0}^{\prime} R_{0} b_{0}+\frac{1}{2} \sum_{i, k=1}^{m} y_{i}^{(0)} \sigma_{i k}^{2} r_{k i}^{(0)} x_{k}^{(0)}
$$

and variance $(2.16)$, where $D_{i k}$ has to be replaced by

$$
D_{i k}-T_{i} D_{i k} T_{k}-T_{i} D_{i k}-D_{i k} T_{k}
$$

and $T_{i}$ is a diagonal matrix consisting of elements $r_{1 i}^{(0)}, \ldots, r_{m i}^{(0)}$ in the diagonal. The same sum that we added to the leading term has to be subtracted from the remainder and it is more realistic to say that the new remainder divided by the dispersion of the new leading term tends stochastically to 0 .

\section{Application to random linear programs}

Consider the linear programming problem

$$
\mu_{0}=\max c_{0}^{\prime} x
$$

subject to the conditions

$$
A_{0} x=b_{0}, \quad x \geq 0
$$

and suppose that it has a unique optimal basis $B_{0}$ which, for the sake of simplicity, we suppose to be the set of vectors $a_{1}^{(0)}, \ldots, a_{m}^{(0)}$. We also suppose that $A_{0}$ has rank $m$. Consider also the problem

$$
\mu=\max c^{\prime} x
$$

subject to the conditions

$$
A x=b, \quad x \geq 0,
$$

where $A, b, c$ have random elements, components, respectively. In these problems we apply the same notations as those used concerning random equations in $\S 2,3$, but we observe that $A$ has $m n$ elements and $c$ has $n$ components.

We suppose also that $B_{0}$ is nondegenerate. There is then a neighborhood of $A_{0}, b_{0}, c_{0}$ in which the problem (4.3)-(4.4) will preserve the subscripts of the optimal basis. Keeping $m$ and $n$ fixed, consider a sequence of random matrices, vectors $A, b, c$, respectively. If we suppose that

$$
\sigma_{\max } \rightarrow 0
$$


where $\sigma_{\max }=\max \left(\sigma_{i k}, t_{i}, s_{k}, i=1, \ldots, m ; k=1, \ldots, n\right)$, the probability that $B=$ $\left(a_{1}, \ldots, a_{m}\right)$ will be the optimal basis to problem (4.3)-(4.4) tends to 1. Hence, according to our lemma, $\left(\mu-\mu_{0}\right) / \sigma$ will have the same asymptotic probability distribution unconditionally or conditionally, given that $B$ is optimal. If, furthermore, conditions (2) and (3) are also satisfied in Theorem 3, where all quantities, vectors, matrices are taken from the random equation $B x=b$, and $c$ means the vector consisting of the first $m$ components of that used in (4.3), then we may state the following.

THEOREM 5 The optimum value $\mu$ of the random programming problem (4.3)-(4.4) has an asymptotic normal distribution with expectation $\mu_{0}$, the optimum of the program taken with the expectations in each place, and variance (2.16), where $x_{0}, y_{0}$ are the primal and dual optimal solutions of the first problem; more exactly, $x_{0}$ is a part of the primal optimal solution consisting of the basic components. The meaning of $D_{i k}, C, F$ remains unchanged. Asymptotic normality means that the probability that $\left(\mu-\mu_{0}\right) / \sigma<x$ tends to $\Phi(x)$.

It is seen from these that the present approach gives a particularly simple result which is very advantageous from the practical point of view because in the characteristics of the random variable $\mu$ such vectors and matrices appear as the primal and dual optimal solutions $x_{0}, y_{0}$ and $D_{i k}, C$ and $F$, the covariance matrices of the random variables involved.

We may also apply Theorem 4 by considering a sequence of programming problems, where $m \rightarrow \infty, n \rightarrow \infty$. Here we suppose that at each problem with $A_{0}, b_{0}, c_{0}$ there is a unique finite, nondegenerated optimum and the probability that the optimal basis has the same column subscripts in problem (4.1)-(4.2) and in (4.3)-(4.4) tends to 1 . Then if we take into account our lemma, the results of Theorem 4 are applicable, where $x_{0}$ and $y_{0}$ have the same meaning as before.

One practical conclusion of these results is the following: if for some reason we solve the linear programming problem with the expectations, e.g., with predicted prices and predicted technology coefficients, but we have information about their random variation, then we may set up confidence limits for the optimum value which would have been the result if we had programmed with the particular realization of the random data in $A, b$, and $c$.

\section{References}

[1] Babbar, M. M. (1955). Distributions of solutions of a set of linear equations (with an application to linear programming). J. Amer. Statist. Assoc., 50, 854-869.

[2] Tintner, G. (1955). Stochastic linear programming with applications to agricultural economics. Second Symposium on Linear Programming, vol. 1, National Bureau of Standards, Washington, D.C., 197-227. 
[3] Tintner, G. (1957). Les programmes linéaires stochastiques. Revue d'Économie Politique, 67, 208-215.

[4] Tintner, G. (1960). A note on stochastic linear programming. Econometrica, 28, 490-495.

[5] Wagner, H. M. (1958). On the distribution of solutions in linear programming problems. J. Amer. Statist. Assoc., 53, 161-163.

[6] Talacko, J. V. (1959). On stochastic linear inequalities. Trabajos Estadíst., 10, 89-112.

[7] Madansky, A. (1960). Inequalities for stochastic linear programming problems. Management Sci., 6, 197-204.

[8] VAJdA, S. (1958). Inequalities in stochastic linear programming. Bull. Inst. Internat. Statist., 36, 357-363.

[9] Kunn, H. W. and R. E. Quandt (1963). An experimental study of the simplex method. Proc. Symp. Appl. Math., 15, 107-124.

[10] Lonseth, A. T. (1942). Systems of linear equations with coeffccients subject to error. Ann. Math. Statist., 13, 332-337.

[11] Lonseth, A. T. (1944). On relative errors in systems of linear equations. Ann. Math. Statist., 15, 323-325.

[12] Lonseth, A. T. (1947). The propagation of errors in linear problems. Trans. Amer. Math. Soc., 62, 193-212.

[13] Box, G. E. P. and J. S. Hunter (1954). A confidence region for the solution of a set of simultaneous equations with an application to experimental design. Biometrika, 41, 190-199.

[14] Quandt, R. E. (1958). Probabilistic errors in the Leontief system. Naval Res. Logist. Quart., 5, 155-170.

[15] Mills, H. D. (1956). Marginal values of matrix games and linear programmes. In: Linear Inequalities and Related Systems, (H. W. Kuhn and A. W. Tucker, eds.), Princeton University Press, Princeton, 183-193.

[16] Williams, A. C. (1963). Marginal values in linear programming. J. Soc. Indust. Appl. Math., 11, 82-94.

[17] Bodewig, E. (1956). Matrix Calculus. North-Holland, Amsterdam.

[18] Cramér, H. (1946). Mathematical Methods of Statistics. Princeton University Press, Princeton.

[19] Gnedenko, B. V. and A. N. Kolmogorov (1954). Limit Distributions for Sums of Independent Random Variables. Addison-Wesley, Reading, Massachusetts. 